\title{
Homonymous Hemianopia with Macular Sparing: Clinical and Radiological Correlation - case presentation
}

\author{
S Baisakhiya, ${ }^{1}$ A Agrawal ${ }^{2}$ \\ ${ }^{1}$ Department of Ophthalmology, Datta Meghe Institute of Medical Sciences, Sawangi (Meghe), Wardha (India) \\ ${ }^{2}$ Department of Surgery, Datta Meghe Institute of Medical Sciences, Sawangi (Meghe), Wardha (India)
}

\begin{abstract}
A classical case of homonymous hemianopia with macular sparing secondary to stroke involving the occipital cortex is presented. The lesion was localized more anteriorly where anatomically the peripheral vision function in the cerebral cortex is located. Sparing of the occipital pole was responsible for macular sparing in the patient. In this case there was good co-relation between clinical symptoms and radiological findings. This case highlights that well conducted visual field testing and careful evaluation of the visual field defect pattern can localize the lesion site approximately along the optic pathway that can be confirmed by modern imaging modalities.
\end{abstract}

Key words: brain, magnetic resonance imaging, macula, occipital lobe, vision, homonymous hemianopia, stroke

\section{INTRODUCTION}

Representation of the visual field in the occipital striate cortex was first delineated by Inouye ${ }^{1}$ and subsequently confirmed by Holmes and Lister. ${ }^{2}$ After the introduction of computerizedd tomography (CT) and magnetic resonance imaging (MRI) of the brain, the accuracy of the Holmes map was confirmed by clinical-radiological correlation. ${ }^{3,4,5}$

\section{CASE REPORT}

A 55-year-old female presented with progressive decrease in peripheral vision on her right side, over a period of one month. It was associated with mild headache that she described more as a discomfort. At the onset of symptoms she had giddiness for 3-4 days. On general and systemic examination she was found to be normal except for a mild rise in blood pressure $(140 / 70 \mathrm{mmHg})$. Higher mental functions were normal and there were no motor or sensory deficits. Visual acuities were R $6 / 18$ and L $6 / 24$ which could be corrected with glasses to $6 / 9$ in both eyes. There were no anterior segment abnormalities. Cover test, pupil reactions and extraocular movements were normal. Fundus examination was normal. Venous pulsations were visible on both optic discs. Intraocular pressures were normal. On confrontation, there were dense right homonymous hemianopic defects affecting both inferior as well as superior visual fields. Automated perimetry (Medmont M600 full screening) confirmed a highly congruous maximum luminance defect in both left hemifields with macular sparing (figure 1). Computerized tomography (CT) showed hypodense nonenhancing lesion in the right occipital cortex (figure 2) that was better seen on MRI (figures 3 and 4). There was also evidence of infarct in left caudate nucleus (figure 5). A diagnosis of occipital lobe infarction was made and she was started on symptomatic treatment.

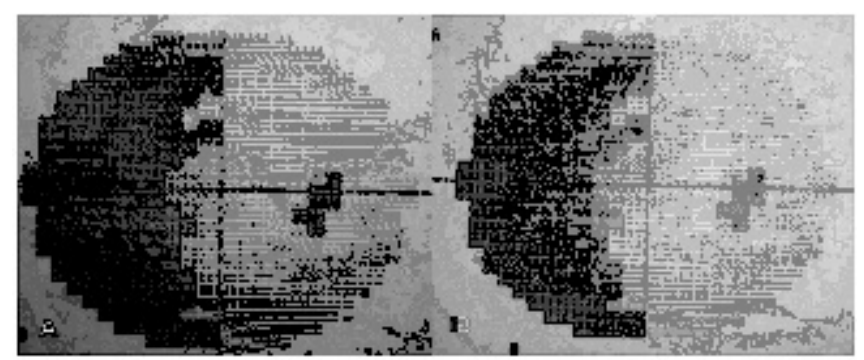

Figure 1. Automated perimetry showing a highly congruous maximum luminance right homonymous hemianopia affecting inferior and superior quadrants

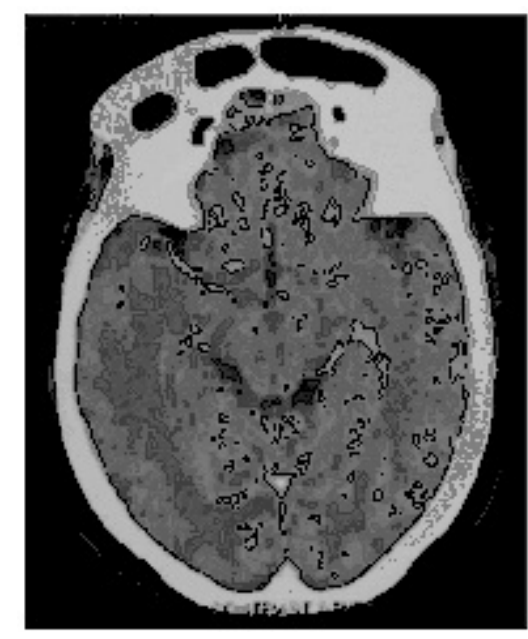

Figure 2. CT scan showing ill-defined hypodense nonenhancing lesion in the right occipital cortex 


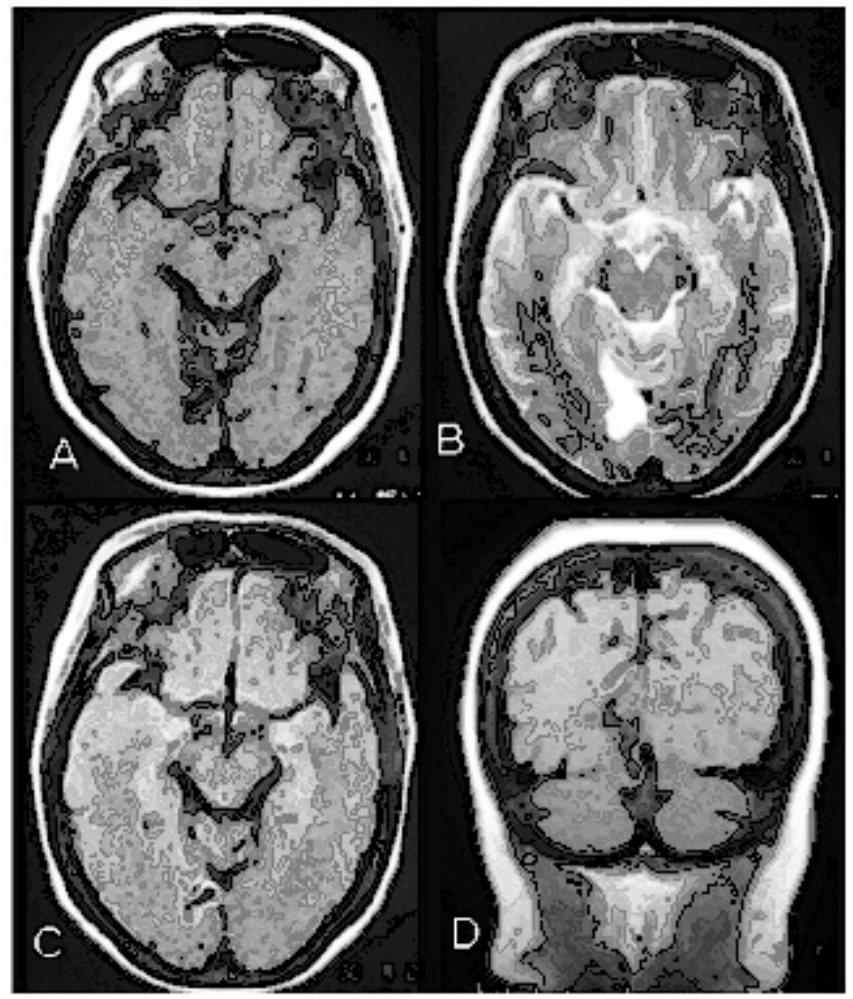

Figure 3. MRI showing details of infarction (A) T1 axial, (B) T2 axial, (C) FLAIR and (D) T 1 coronal image

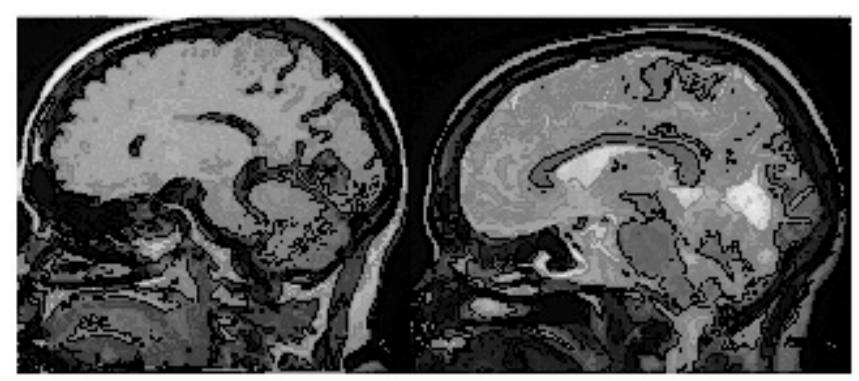

Figure 4. MRI sagittal sections showing details of infarction (A) T1, (B) T2 images

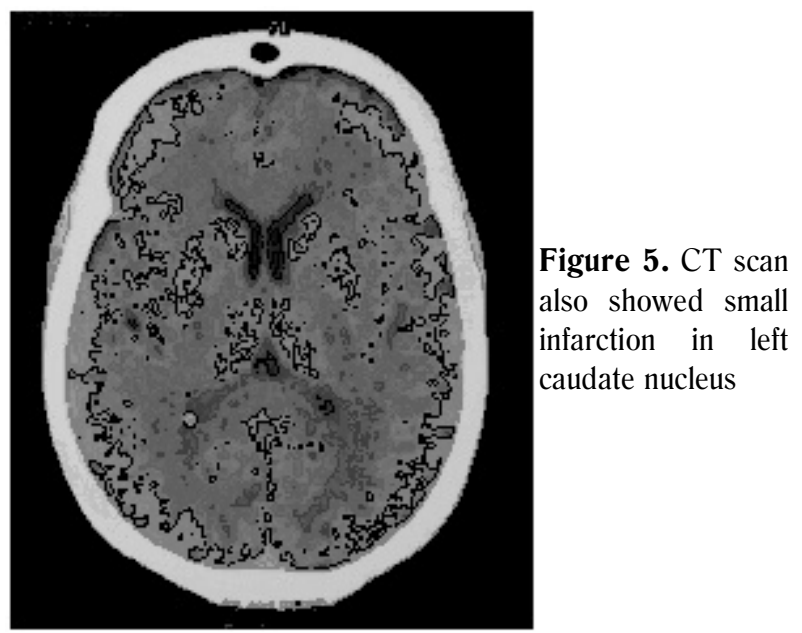

\section{DISCUSSION}

Common causes of homonymous hemianopia include mass lesions, intracranial haemorrhage or infarction in the territory of major cerebral arteries, trauma or rarely complicated migraine. ${ }^{6}$ The representation of the field of vision in the human striate cortex is based on the Holmes map in which about 25\% of the surface area of the striate cortex is allocated to the central 15 degrees of vision. ${ }^{5,7}$ The foveal representation is located at the occipital pole, where the striate cortex usually extends about $1 \mathrm{~cm}$ onto the lateral convexity of the occipital lobe. The extreme periphery of the visual field is represented anteriorly, at the junction of the calcarine and parietooccipital fissures. ${ }^{7}$ Macular sparing is associated with survival of the occipital pole in some instances. ${ }^{3,5}$ In this study, the lesion was localized more anteriorly where the cortex is responsible for peripheral vision. Sparing of the occipital pole (by the haemorrhage) was responsible for macular sparing. In this patient, there was good co-relation between clinical symptoms and radiological findings. This case illustrates the clinical value of confrontation and visual field testing. Well-conducted visual field testing and careful evaluation of the visual field defect pattern can localize the lesion site approximately along the optic pathway that can be confirmed by modern imaging modalities.

\section{REFERENCES}

1. Inouye T. Die Sehstorungen bei Schussverletzengen der kortikalen Sehsphare. Leipzig, Germany: Engelmann; 1909

2. Holmes G, Lister WT. Disturbances of vision from cerebral lesions with special reference to the cortical representation of the macula. Brain 1916; 39: 34-73.

3. McAuley DL, Russell RW. Correlation of CAT scan and visual field defects in vascular lesions of the posterior visual pathways. J Neurol Neurosurg Psychiatry 1979; 42(4): 298-311.

4. Kattah JC, Dennis P, Kolsky MP, Schellinger D, Cohan SL. Computed tomography in patients with homonymous visual field defects: a clinico-radiologic correlation. Comput Tomogr 1981; 31: 1098-1106.

5. McFadzean R, Brosnahan D, Hadley D, Mutlukan E. Representation of the visual field in the occipital striate cortex. Br J Ophthalmol $1994 ; 78(3): 185-90$.

6. Miller NR. Walsh and Hoyt's Clinical Neuro-Ophthalmology, 4th ed. Baltimore: Williams \& Wilkins; 1988.

7. Korogi Y, Takahashi M, Hirai T, Ikushima I, Kitajima M, Sugahara T, Shigematsu Y, Okajima T, Mukuno K. Representation of the visual field in the striate cortex: comparison of MR findings with visual field deficits in organic mercury poisoning (Minamata Disease). AJNR Am J Neuroradiol 1997; 18: 1127-1130. 